\title{
Fuzzy based Channel Selection for Location Oriented Services in Multichannel VCPS Environments
}

\author{
Reena Kasana, Member IEEE, Sushil Kumar, Member IEEE, Omprakash Kaiwartya, Member IEEE, Rupak Kharel, \\ Member IEEE, Jaime Lloret, Senior Member IEEE, Nauman Aslam, Member IEEE, Tong Wang
}

\begin{abstract}
Location-oriented services in Vehicular CyberPhysical System (VCPS) have witnessed significant attention due to their potentiality to address traffic safety and efficiency related issues. The multichannel communication aids these services by tuning their overall performance in vehicular environments. Related literature on multichannel communication is focuses on interference as channel quality measure. However, uncertain mobility and density of vehicles significantly affect channel quality apart from interference. The static quantification of channel quality is not suitable due to the dynamic characteristics of the channel quality parameters. In this context, this paper proposes Fuzzy-based Channel Selection framework for location-oriented services in Multichannel VCPS environments (F-CSMV). A system model is presented for deriving channel access delay using Markov chain model. The channel quality is estimated using channel access delay (CAD) and signal-to-interference ratio (SIR). The fuzzy logic based channel selection framework is developed considering fuzzification and defuzzification of CAD and SIR. The comparative performance evaluation attests the benefit of the framework as compared to the state-of-the-art techniques in VCPS.
\end{abstract}

Index Terms-Location service, Vehicular network, Fuzzy, VCPS

\section{INTRODUCTION}

$\mathrm{T}$ he technological development in the field of Vehicular Adhoc Networks (VANETs) and Cyber-Physical Systems (CPS) has attracted the attention of academic researchers and practitioners in the design, and development of Vehicular Cyber-Physical Systems (VCPS) [1, 2]. VANETs enable data communication between vehicles in a distributed manner in VCPS. It is a promising technology enabling a myriad of location oriented safety and comfort applications [3, 4]. The availability of low-cost GPS receiver has led to the wide-spread adoption of location-oriented services in intelligent transportation systems [5, 6]. The IEEE 802.11p working group has developed Wireless Access in Vehicular Environment (WAVE) standard implementing a set of quality of support modules for location-oriented services [7].

The research is supported by the gLINK project at the Northumbria University UK (EU grant No: 2014-0861/001-001- EM Action 2- Partnerships).

R. Kasana and S. Kumar are with Jawaharlal Nehru University (JNU), New Delhi, 110067, India. Email: \{skdohare, reena24_scs\}@jnu.ac.in

O. Kaiwartya (Corresponding Author) and N. Aslam with the Northumbria University, Newcastle, NE1 8ST, UK. Email: Omprakash.kaiwartya; nauman.aslam@northumbria.ac.uk

R. Kharel is with the School of Engineering, Manchester Metropolitan University, M1 5GD, UK Email: r.kharel@mmu.ac.uk

J. Lloret is with the Universitat Politècnica de València, 46022, Valencia, Spain. Email: jlloret@ dcom.upv.es

T. W. is with the Harbin Engineering University, Harbin, 150001, China. Wang.tong@hrbeu.edu.cn
The federal communication commission of the United States allocated $75 \mathrm{MHz}$ of bandwidth in the frequency band $5.850-$ $5.925 \mathrm{GHz}$ as dedicated short range communications spectrum in order to accommodate vehicle-to-vehicle $(\mathrm{V} 2 \mathrm{~V})$ and vehicleto-infrastructure (V2I) communications. Out of the available bandwidth, $5 \mathrm{MHz}$ is reserved as the guard band, and rest is divided into seven non-overlapping channels. These seven channels are numbered in the range $172-184$. The bandwidth assigned to each channel is $10 \mathrm{MHz}$. The channel 178 is designated as Control Channel ( $\mathrm{CCH})$ and dedicatedly used for transmitting control messages or network management data. The remaining channels are referred as Service Channels (SCHs) used for non-critical data transmission including traffic, infotainment, Internet services and location-related data [8]. The IEEE 1609.4 standard is a part of the WAVE stack architecture [9]. It provides the capability of multichannel operation to the IEEE 802.11p Medium Access Control (MAC) protocol. The multichannel operation in vehicular environments defines a number of channels due to which the vehicles in the same geographical region can transmit traffic information over the multiple channels simultaneously. There are number of benefits of the multi-channel operation in vehicular environments including better utilization of bandwidth favoring application which require high data rate, higher throughput due to the parallel transmissions, and enhanced robustness in transmission in the presence of interference, and noise [10].

The protocols supporting multichannel operations intelligently select channels for enhancing bandwidth utilization by avoiding channels with high level of perturbations. The multichannel communication in vehicular environments can be enabled using the dual-radio transceiver. Although the initial release of vehicular communication systems was based on single-radio transceiver in vehicles yet, the next generation vehicular communication systems will rely on dual-radio transceiver settings ensuring better performance. In vehicular environments, vehicles have the option to use any of the six SCHs for data transmission. The real-time trafficoriented selection of appropriate channel for data transmission is challenging task in multichannel vehicular environments. IEEE 1609.4 and European telecommunications standards institute specifications suggest selecting the least congested channel for data transmission [10]. However, it is not mentioned in the specifications how to select the least congested channel, as quantifying channel congestion is complex in dynamic vehicular environments. The literature on multichannel communication in vehicular environments is based on the received signal-to-interference ratio (SIR) as the channel quality measure $[11,12]$. However, SIR-based static quantification of channel quality might lead to the selection of 
a congested channel in multichannel vehicular environments. It is due to the uncertain vehicles mobility and density significantly affecting channel quality apart from the interference in vehicular environments[11]. The static quantification of channel quality is unable to incorporate the impact of vehicular environments considering the dynamic characteristics of the parameters directly affecting channel quality. Moreover, fuzzy-based techniques have been effectively applied for the quantification of parameters in dynamic environments in various domains [13].

In this context, this paper proposes a Fuzzy-based Channel Selection framework for location-oriented services in Multichannel Vehicular cyber-physical system environments (F-CSMV). The framework aids the location-oriented ITS application by enabling vehicles to appropriately select transmission channel for better bandwidth utilization in multichannel vehicular environments. In particular, the channel selection framework can be defined in four major folds as contributions of the paper.

1) Firstly, a system model is presented for deriving channel access delay using Markov Chain model to define channel states in multichannel vehicular environments.

2) Secondly, estimation of two channel quality parameters namely, channel access delay (CAD) and signal-tointerference ratio (SIR) is carried out using self-adaptive spectrum and combined shadowing and path loss, respectively.

3) Thirdly, fuzzy logic-based channel selection framework is developed focusing on fuzzification and defuzzification of channel quality parameters including CAD and SIR.

4) Finally, the framework is tested to comparatively evaluate the performance with state-of-the-art techniques considering channel and network performance related metrics in vehicular network environments.

\section{RELATED WORK}

This section briefly reviews channel selection in multichannel environments focusing on the single transceiver and multi-transceiver based techniques.

\section{A. Single-transceiver based Multichannel Communication}

Vehicular MESH (VMESH)[14] has been suggested to address the drawbacks of IEEE 802.11 DCF and EDCA in supporting non-safety applications which are throughputsensitive in a multi-channel vehicular environment. However, the $\mathrm{CCH}$ period in VMESH has been further divided into two parts which reduces the transmission opportunities of safety messages. VeMAC [15] is a TDMA-based MAC protocol and has been designed for the multi-channel vehicular environment to achieve reliable and efficient one-hop as well as multi-hop broadcast services on the $\mathrm{CCH}$ without hidden terminal problem. However, time slots wastage may occur in case of not enough nodes in a neighborhood to use all the time slots of a frame [16]. Multi-schedule-based channel switching (MSCS) protocol has been suggested for efficient utilization of scarce spectrum resources [17]. In MSCS, choice of SCHs depends on the application for which vehicle wants to utilize the channel. An RSU-coordinated synchronous MAC protocol has been presented for multichannel VANETs which reduces congestion on $\mathrm{CCH}$ as well as solves the multi-channel hidden terminal problem [18]. In addition, RSUs has been utilized to store rendezvous information and transmit it to moving vehicles. An adaptive multi-channel assignment protocol utilizing real-time traffic condition has been suggested [19]. Channel switching interval has been adjusted on the basis of congestion level measured in real-time.

\section{B. Multi-transceiver based Multichannel Communication}

Dynamic channel allocation (DCA) scheme has been suggested to assigns channels on-demand [20]. The available bandwidth is divided into $n$ data channel and one control channel. The control channel has been utilized to solve the problem of contention among data channels. For data transmission, nodes choose the first detected idle channel without taking channel quality into consideration. Therefore, this approach fails in vehicular environment due to timevarying channel impairments and contention fluctuations. DCA has been enhanced to address power control problems along with dynamic channel-assignment and multiple-channel access using power control (DCA-PC) mechanism [21]. This protocol send control packets on the $\mathrm{CCH}$ with maximum transmission power whereas the data channels are used with suitable power control for exploiting channel reuse. This protocol achieve better throughput than DCA. However, the effect of power control can be seen only with limited number of channels. The $\mathrm{CCH}$ becomes overloaded with the increase in number of channels.

A receiver-based distributed channel selection protocol has been suggested for maximum bandwidth utilization [12]. The protocol intelligently selects best channel for data transmission on the basis of signal-to-interference-plus-noise ratio (SINR). A multichannel MAC protocol for multiple access (MMA) in vehicular environment has been suggested for increasing channel efficiency [22]. A clustering-based multichannel MAC protocol supporting QoS requirements of multimedia and data applications has been suggested [23]. A cluster head utilizes IEEE 802.11p CSMA/CA mechanism to contend for the medium on a different frequency and cluster members use TDMA. A cross-layer approach has been presented for dynamically switching channels in a multichannel multi-radio environments (IAR) [11]. An interference-aware metric has been defined to alleviate the impact of co-channel interference perceived by a vehicular node. This metric is used at the network layer and tries to maximize the average SIR level of the path between the source and the destination vehicle. Service Actuated Multi-Channel operation (SAMCO) has been suggested for dynamic channel switching and service prioritization [24, 25]. Metrics such as user preference, channel load, and services on the channel have been utilized in channel selection algorithm.

\section{FUZZY BASED CHANNEL SELECTION FRAMEWORK}

In this section, the detail of the fuzzy logic based channel selection framework F-CSMV is presented. It focuses on system model for deriving Markov chain based channel states in multichannel environments, estimation of channel quality parameters including channel access delay and signal-to- 
interference ratio and fuzzy-based framework for channel selection.

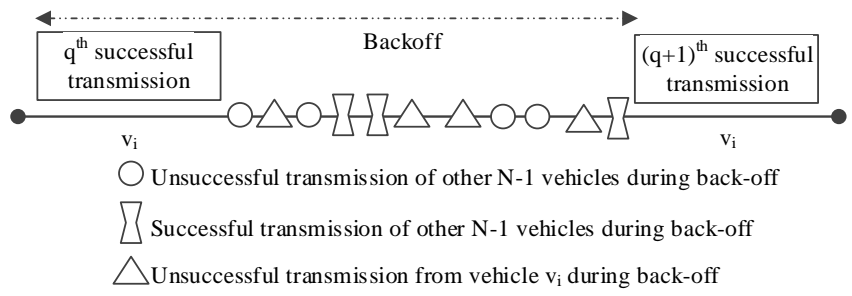

Fig. 1. Channel access delay in multichannel vehicular environments

\section{A. System Model}

The channel access delay in multichannel environments is defined as the interval between the time a data packet reaches the head of the transmission queue and begins contending for the channel, and the time of successful reception of the packet at the receiver. In vehicular environments, the modified version of listen-before-transmit channel access scheme is considered for avoiding collision of packets. A vehicle cannot start transmission immediately, even if a channel is idle. The neighboring vehicles may successfully transmit a number of packets or may be involved in a number of collisions between $q^{t h}$ and $(q+1)^{t h}$ successful transmissions of a vehicle $v_{i}$, each of which is added to the channel access time of the vehicle (see Fig. 1). The transmissions attempted by vehicle $v_{i}$ and resulting in collisions are also included in this channel access time. The channel access delay comprises of components including deferring transmission time in case of busy channel, DIFS, random duration of transmission deferring for collision reduction, and delayed transmission due to the collision. The channel access delay in multichannel vehicular environment can be defined using Markov chain based derivation for identifying channel states [26]. The channel state Markov chain accurately describes channel state in multichannel environments while the tagged vehicle is in back-off mode. The modelling of Tagged Vehicle Markov chain (TVMC) and Channel State Markov Chain (CSMC) are detailed below:

\section{1) Tagged Vehicle Markov Chain}

Let $B(t)$ and $S(t)$ are two stochastic processes representing backoff counter and backoff stage $j$ at time $t$, respectively which form the tagged vehicle markov chain. The value of $B(t)$ is uniformly distributed in the range $\left(0,1 \ldots W_{j}-1\right)$ where $W_{j}$ can be determined as given by Eq. (1).

$$
W_{j}= \begin{cases}2^{j} C W_{\min } & \text { if } 0 \leq j<m \\ 2^{m} C W_{\min } & \text { if } m \leq j \leq L\end{cases}
$$

where, $C W_{\min }$ and $C W_{\max }$ are the size of minimum and maximum contention window, $m=\log _{2}\left(\frac{C W_{\max }}{C W_{\min }}\right)$ is the maximum backoff stage, and $L+1$ is the maximum number of retransmissions limit before dropping a packet. Thus, the value of backoff stage $S(t)$ lies in the range $(0,1 \ldots L+1)$. By using Eq. (1), the probability of transmission attempt in the multichannel vehicular environment can be expressed as given by Eq. (2).

$$
\tau=\frac{1-P^{L+1}}{\left(\sum_{j=0}^{L}\left[1+\frac{1}{1-P_{f}} \sum_{k=1}^{W_{j}-1 W_{j^{-1}}} \frac{W_{j}}{P^{j}}\right)(1-P)\right.}
$$

where $P_{f}$ is the freezing probability. The conditional collision probability $P$ that a tagged vehicle sees a transmission originated by at least one of the other contending vehicles can be expressed as given by Eq. (3).

$$
P=1-(1-\tau)^{N-1}
$$

The freezing probability $P_{f}$ can be accurately calculated using channel state which is derived in the next section.

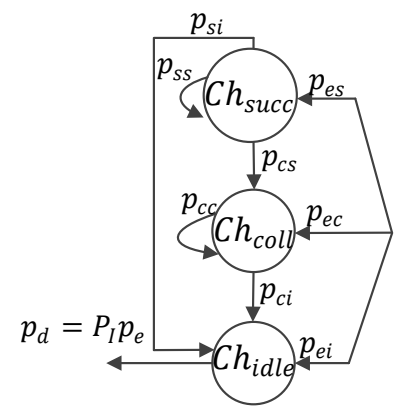

Fig. 2: Multichannel Vehicular Environments as Channel State Markov Chain

\section{2) Channel State Markov Chain}

In multichannel vehicular environments, a channel can be in any of the three states including $C h_{\text {succ }}, C h_{\text {coll }}$, and $C h_{\text {idle }}$, representing a successful transmission, collision and idle states, respectively during the tagged vehicle backoff period (see Fig. 2). Consequently, the steady state probability of channel states is represented by $P_{C h_{s u c c}}, P_{C h_{\text {coll }}}$ and $P_{C h_{\text {idle }}}$, respectively. In realistic vehicular environments, a backoff state can be entered from either a transmission state or from a previous back-off state. The probability $p_{e i}$ of entering into backoff state and then finding the channel idle by a tagged vehicle can be expressed as given by Eq. (4).

$$
p_{e i}=(1-\tau)^{N-1}
$$

Similarly, the probability of entering in back-off state and finding the channel busy after a successful transmission $p_{e s}$ are and collision $p_{e c}$ can be expressed as given by Eq. (5) and (6).

$$
\begin{aligned}
& p_{e s}=\left(\begin{array}{c}
N-1 \\
1
\end{array}\right) \tau(1-\tau)^{N-2} \\
& p_{e c}=1-p_{e i}-p_{e s}
\end{aligned}
$$

The two other scenarios are considered for calculating other transition probabilities between the channel states (see Fig. 2). In one scenario, the tagged vehicle enters in back-off state and observes the channel to be in the successful transmission state. In this case, two possibilities are there. Firstly, the tagged vehicle observes successive successful transmissions only when the vehicle that successfully transmitted a packet selects the new back-off counter as zero, and the remaining vehicles do not carry out transmissions. In that case, the channel remains in the state $C h_{s u c c}$ with probabilities $p_{s s}$. Secondly, vehicle with successfully transmitted packets select a non-zero back-off counter then the channel state becomes $C h_{\text {idle }}$ with probability $p_{s i}$. Therefore, the successive transition probability $p_{s s}$ and $p_{s i}$ can be expressed as given by Eq. (7) and (8). 


$$
\begin{aligned}
& p_{s s}=\frac{1}{W_{0}} \\
& p_{s i}=1-p_{s s}
\end{aligned}
$$

In the second scenario, the tagged vehicle enters in back-off state and observes the channel in collision state. The channel moves from collision to successful transmission state in the next time slot if all the non-colliding vehicles do not transmit in that slot. Considering $n$ number of vehicles in collision state, the probability distribution that only $n$ vehicles among the $N$ vehicles participate in the last transmission can be computed as given by Eq. (9).

$$
Q(n)=\left(\begin{array}{c}
N-1 \\
n
\end{array}\right) \tau^{n}(1-\tau)^{N-n-1}
$$

The transition probability from collision state to idle state $p_{c i}$ and from collision to success state $p_{c s}$ and remain in collision state $p_{c c}$ can be calculated as given by Eq. (10), (11), and (12).

$$
\begin{gathered}
p_{c i}=\sum_{n=2}^{N-1} Q(n)\left(1-\frac{1}{\overline{c W}}\right)^{n} \\
p_{c s}=\sum_{n=2}^{N-1} Q(n) n\left(\frac{1}{\overline{c W}}\right)\left(1-\frac{1}{\overline{c W}}\right)^{n} \\
p_{c c}=1-p_{c i}-p_{c s}
\end{gathered}
$$

By using all the transmission probabilities, the steady-state probabilities of the channel state vector $A=$ $\left[\begin{array}{lll}P_{\text {Ch }} \text { succ } & P_{C h_{\text {coll }}} & P_{C h_{\text {idle }}}\end{array}\right]$ are obtained by solving Eq. (13).

$$
T A=A
$$

where $T=\left[\begin{array}{ccc}p_{e i} & p_{e s} & p_{e c} \\ p_{s i} & p_{s s} & 0 \\ p_{c i} & p_{c s} & p_{c c}\end{array}\right]$ represents the transition probability matrix of CSMC. By considering the above states of channel, the freezing probability $P_{f}$ can be expressed as Eq. (14).

$$
P_{f}=1-P_{d}=1-P_{\text {Chidle }}
$$

An iterative approach is considered for computing the steadystate probabilities in TVMC and CSMC. The iterative steps are summarized in Fig. 3 where the transmission probability for the next round is computed by using exponentially weighted moving average and smoothening factor $\beta$ as expressed by Eq. (15).

$$
\tau^{(1)}=\beta \tau^{(0)}+(1-\beta) \tau_{n e w}
$$

The value $\beta=0.5$ is considered to give equal importance, i.e., $50 \%$ to the most recent observation and the next observation. The value $\varepsilon=0.0001$ is considered towards higher precision, as smaller value of $\varepsilon$ improves the precision in the related calculation of the parameter $\tau$.

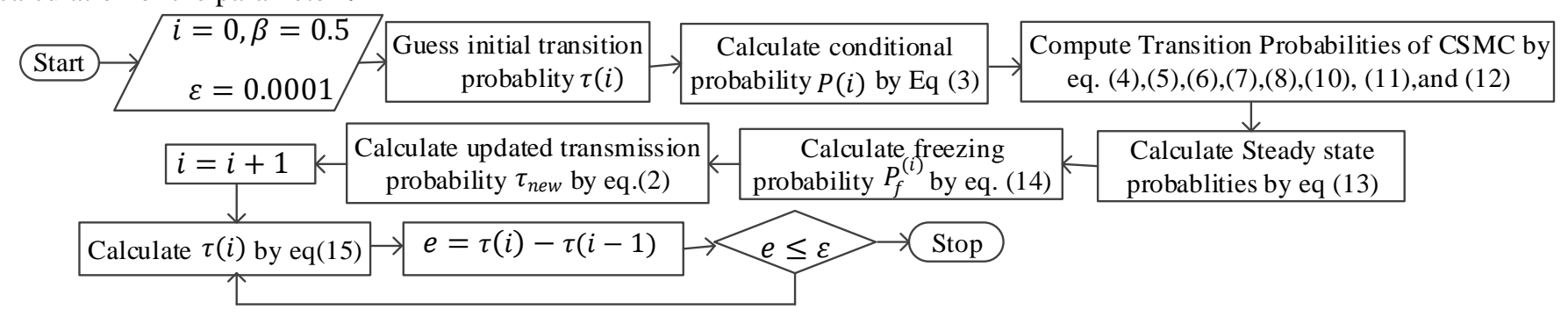

\section{B. Estimation of CAD and SIR}

The two channel quality parameters CAD and SIR are quantified for multichannel vehicular environments.

\section{1) Channel Access Delay}

The self-adaptive spectrum (SAS) is considered to estimate the number of contending vehicles for transmitting on a particular channel in multichannel vehicular environments [12]. It is a management middleware for wireless network which performs dynamic estimation of traffic load $L\left(C h_{c}\right)$, in multichannel network environments. The number of contending vehicles $N\left(C h_{c}\right)$ for a channel $C h_{c}$ in multichannel environments can be estimated as given by Eq. (16).

$$
N\left(C h_{c}\right)=\operatorname{ceil}\left(-H\left(C h_{c}\right) \times \ln \left(1-L\left(C h_{c}\right)\right)\right)
$$

where $H\left(C h_{c}\right)$ represents the $H$ time slots on channel $C h_{c}$. After estimating $N\left(C h_{c}\right)$, average $C A D\left(C h_{c}\right)$ for channel $C h_{c}$ is calculated. Let $\mathcal{F}$ be the average duration a tagged vehicle remains in a backoff stage before decrementing its backoff counter and is calculated as expressed by Eq. (17).

$$
\mathcal{F}=\left(p_{e i} D_{i c h}+p_{e s} D_{s c h}+p_{e c} D_{c c h}\right)\left(\frac{1-\tau}{1-P_{f}}+\frac{\tau(\overline{C W}-1)}{\overline{C W}}\right)
$$

where, $\overline{C W}$ is the average contention window size for all backoff stages, and $D_{i c h}, D_{s c h}$, and $D_{c c h}$ are the time duration that a tagged vehicle spends in a single backoff state, when the state of the channel at the time the vehicle enters into the backoff state is idle, busy with successful transmission and busy with collision, respectively.

For deriving the value of $D_{i c h}, D_{s c h}$, and $D_{c c h}$, three situations are considered. In first scenario, when a tagged vehicle enters into a back-off state, and finds the channel idle, then the vehicle decrements the back-off counter after waiting for 1 time-slot and then leave the current back-off state. Hence, $D_{i c h}=1$. In another scenario, when a tagged vehicle enters into a back-off state, and the channel is busy with the successful transmission. The tagged vehicle waits in the back-off state for the time duration of successful transmission and any other successive successful transmissions and one additional idle slot at the end. The probability of successive successful transmission is equal to $p_{s s}=1 / W_{0}$, and the average number of successive successful transmission is $\frac{1}{1-p_{s s}}$. Hence, $D_{s c h}$ can be expressed as given by Eq. (18).

$$
D_{s c h}=\frac{1}{1-p_{s s}} T_{s}+D_{i c h}
$$


In the third scenario, a tagged vehicle enters into a back-off state, and the channel is busy with a collision. The tagged vehicle waits in the back-off state for the time duration of present collision followed by any collision or successful transmission, and one additional idle slot at the end. The average number of successive collisions can be $\sum_{i=0}^{L} i p_{c c}^{i}$ in the network. After a collision, either one successful transmission followed by an idle slot with probability $\frac{p_{c s}}{1-p_{c c}}$, or an idle slot with probability $\frac{p_{c i}}{1-p_{c c}}$ happens. Hence, $D_{c c h}$ can be computed as given by Eq. (19).

$$
D_{c c h}=\left(\sum_{i=0}^{L} i p_{c c}^{i}\right) T_{c}+\frac{p_{c s}}{1-p_{c c}} D_{s c h}+\frac{p_{c i}}{1-p_{c c}} D_{i c h}
$$

The average $C A D\left(C h_{c}\right)$ for given channel $C h_{c}$ in multichannel vehicular environments is computed as expressed by Eq. (20).

$$
C A D\left(C h_{c}\right)=\frac{1}{1-P_{\text {drop }}} \sum_{i=0}^{L} P_{\text {succ }}^{(i)}\left[T_{s}+i T_{c}+\left(\sum_{j=0}^{i} \bar{W}_{j} \mathcal{F}\right)\right]
$$

where $P_{\text {drop }}=P^{L+1}$ is the probability of dropping a packet after $L+1$ transmissions, $P_{\text {succ }}^{(i)}=(1-P) P^{i}$ is the probability of successful transmission after $i$ number of retransmissions, $\bar{W}_{j}=\left(W_{j}-1\right) / 2$ represent average number of back-off slots at stage $j, T_{s}$ is the average duration of successful transmissions, and $T_{c}$ is the average collision duration. For basic transmission mode, $E\left[T_{s}\right]=E\left[T_{c}\right]=D I F S+T_{h}+T_{p}+$ $S I F S+T_{A C K}$ represents average $T_{S}$ and $T_{c}$, where $T_{h}$ and $T_{p}$ is the transmission duration of medium access and physical layer headers.

\section{2) Signal-to-Interference Ratio}

In multichannel vehicular environments, SIR is derived considering the combined impact of shadowing and path loss on the received signal. The received power $P_{i j}^{r}$ at a vehicle $V_{j} \in$ $n b_{\text {list }}\left(V_{i}\right)$ due to the transmissions of vehicle $V_{i}$ on its own channel can be expressed as given by Eq. (21).

$$
P_{i j}^{r}=P_{i}^{t} \times K \times r_{i j}^{-\eta} \times X_{i j}
$$

where, $P_{i}^{t}$ is the transmitted power by $V_{i}, \mathrm{~K}$ is the constant representing channel attenuation and antenna characteristics, $r_{i j}$ is the distance between $V_{i}$ and $V_{j}, \eta$ is the path loss exponent, $X_{i j}=10^{\xi_{i j} / 10}$ is a lognormal random variable characterizing shadowing, and $\xi_{i j}$ is a normal distributed random variable with zero mean and standard deviation $\sigma$. The received power $P_{m j}^{r}\left(C h_{c}\right)$ at a vehicle $V_{j}$ on channel $C h_{c}$ due to the simultaneous transmissions by a set of interfering vehicles $V_{m}=\left\{V_{1}, V_{2} \ldots V_{M}\right\}$ can be expressed as given by Eq. (22).

$$
P_{m j}^{r}\left(C h_{c}\right)=\sum_{i=1}^{M} P_{m}^{t} K \cdot r_{i j}^{-\eta} X_{m j}
$$

By using Eq. (21) and (22), SIR in multichannel vehicular environments is determined by the ratio of the desired signal power to the total interference power from all the other vehicles, as expressed by Eq. (23).

$$
S I R_{i, C h_{c}}=\frac{P_{i j}^{r}}{\sum m \neq i_{m j}^{r}\left(C h_{c}\right)}
$$

\section{3) Fuzzy Logic based Channel Selection}

The fuzzy logic-based channel selection framework FCSMV is designed to enable a vehicle to decide intelligently whether the channel switching procedure should be triggered or not. The framework operates locally at each vehicular node and controls the channel switching procedure, using linguistic rules that describe the behavior of the channel. It implements a nonlinear decision probability to trigger the decision and uses the instantaneous channel quality parameters, CAD and SIR.

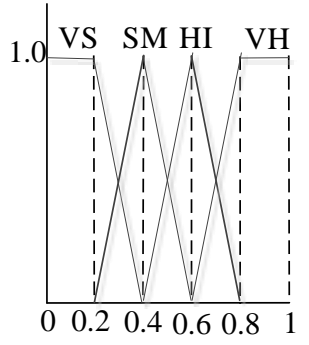

Channel Access Delay

(a)

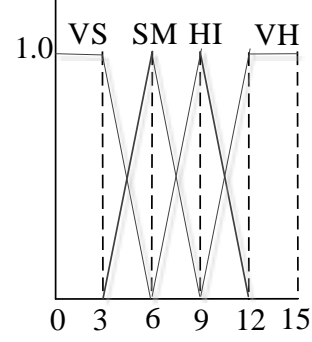

Signal to Interference Ratio

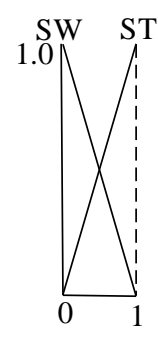

Result

(c)
Fig. 4. The fuzzy membership functions for (a) CAD, (b) SIR, (c) output

It is to be highlighted that there are various factors affecting channel state including network load, communication range, node distance, bandwidth availability, contention window size, and physical obstruction. The proposed work considers two parameters, namely CAD and SIR for estimation of channel state. These two parameters directly or indirectly consider most of the mentioned factors affecting the channel state and hence, suitable for the design of an efficient channel switching algorithm for multichannel vehicular environments. The average $\mathrm{CAD}$ is considered as a metric to identify and measure network congestion in real-time whereas SIR perceived by the receiver is considered as a link quality parameter. Solely using one metric for channel selection may induce vehicles to use a channel which is more congested. A channel selection scheme should take both the link quality and level of congestion into account to decide which channel to use and when. The fuzzy membership functions are defined as shown in Fig 4. The channel-quality parameter value range is divided into four labels. The quantification of labels of the fuzzy variables is defined in Eq. (24).

$$
\text { Fuzzy Quantification }=\left\{\begin{array}{c}
C A D \\
S I R
\end{array}(V S, S M, H I, V H)\right.
$$

where $V S, S M, H I$, and $V H$ are the defined value range representing very small, small, high, and very high fuzzy parameter values. There are four value range divisions for CAD with equal length of 0.2 , and similarly, four value range for SIR of length 3 . These value range are deterministic and can be different for other fuzzy based channel selection system. The decrement in value range increases complexity of fuzzy system and reduces responsiveness. These four labels of both the channel quality parameters determine the fuzzy output value which is converted as $R S T$ (result) $=\{S W$ or ST), where SW represents switch the channel and ST denotes stay in the channel. The sixteen combination of fuzzy if-then rules are presented in Table I. The channel selection procedure at a vehicular node is triggered each time any sender vehicle intends to transmit a data packet. After selecting a channel, the transmitting vehicle routes a service advertisement message 


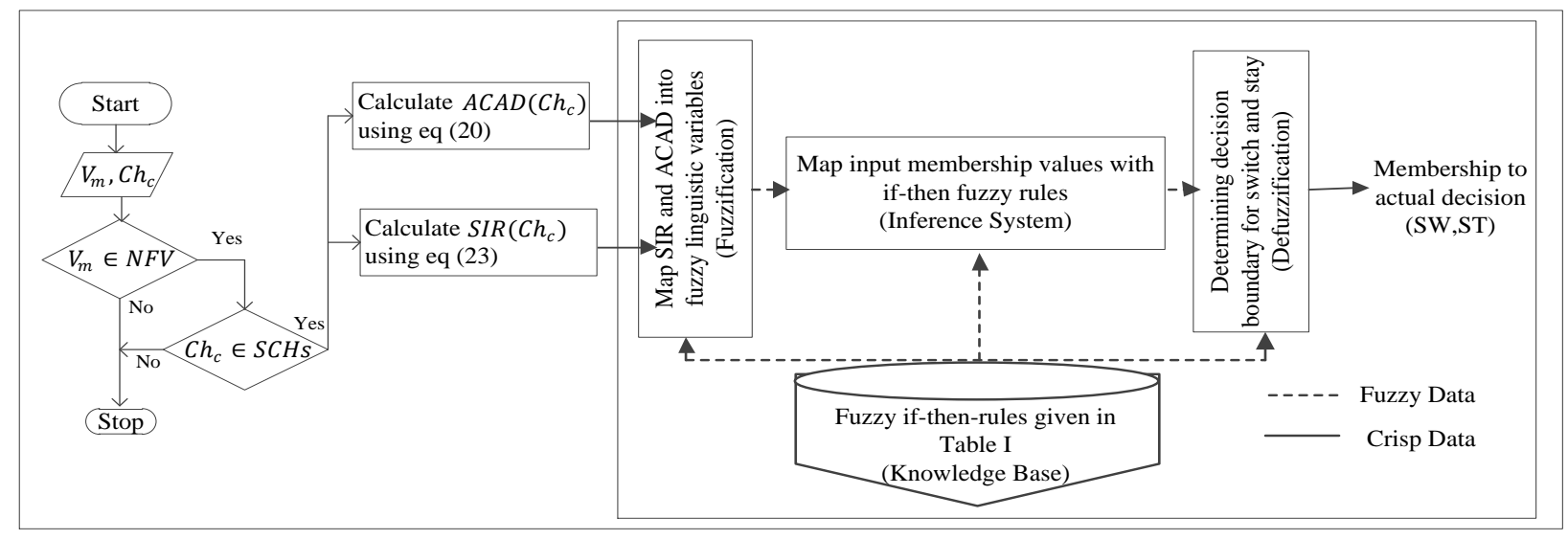

Fig. 5. Fuzzy-based channel selection framework

internally to the intended channel and the receiving vehicle tunes to the right channel, so that it can receive the packet. The block diagram of the fuzzy based channel selection framework is presented in Fig.5

Table I. Fuzzy if-then rules for channel selection

\begin{tabular}{cccccccc}
\hline \multirow{2}{*}{ Rule } & IF & & THEN & Rule & IF & & THEN \\
\cline { 2 - 3 } & CAD & SIR & RST & & CAD & SIR & RST \\
\hline 0 & VS & VS & SW & 8 & HI & VS & SW \\
1 & VS & SM & ST & 9 & HI & SM & SW \\
2 & VS & HI & ST & 10 & HI & HI & SW \\
3 & VS & VH & ST & 11 & HI & VH & ST \\
4 & SM & VS & SW & 12 & VH & VS & SW \\
5 & SM & SM & SW & 13 & VH & SM & SW \\
6 & SM & HI & ST & 14 & VH & HI & SW \\
7 & SM & VH & ST & 15 & VH & VH & SW \\
\hline
\end{tabular}

\section{EMPIRICAL RESULTS}

In this section, analytical and simulation-based results are discussed for analyzing the performance of the proposed Fuzzybased Channel Selection framework. This section is broadly divided into two parts. In the first part, analytical results are discussed, whereas simulation setting, performance metrics and comparative analysis of simulation results are discussed in the second part.

\section{A. Analytical Results}

The analytical analysis evaluates the performance of the mathematical formulations used for selection of service channel in VCPS environment using a mathematical tool. The average CAD has been measured by varying various parameters including number of vehicles $(N)$, minimum contention window size $\left(C W_{\text {min }}\right)$ and bandwidth of the channel $(B W)$.

The impact of varying $N$ and $C W_{\min }$ on $A C A D$ experienced by a vehicle on a channel is shown in Fig. 6(a). It can be clearly observed that $A C A D$ increases with increase in $N$. This is because of the fact that with increase in $N$, number of collisions as well as waiting time to access the $\mathrm{SCH}$ increases, in turn increasing $\mathrm{ACAD}$. For low values of $N$, ACAD decreases with increase in $C W_{\text {min }}$. However, for higher values of $N$, ACAD first increases and then starts decreasing with increase in $C W_{\text {min }}$. This can be attributed to the fact that for smaller $C W_{\min }$ and higher $N$, system reaches saturation state and cannot further serve incoming packets.

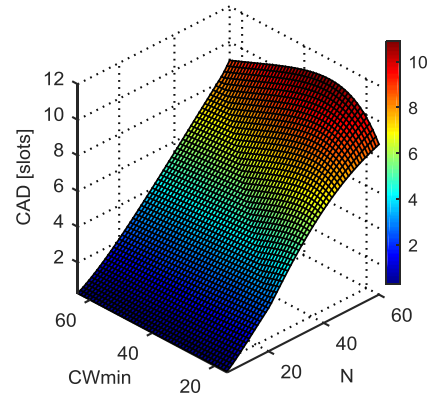

(a)

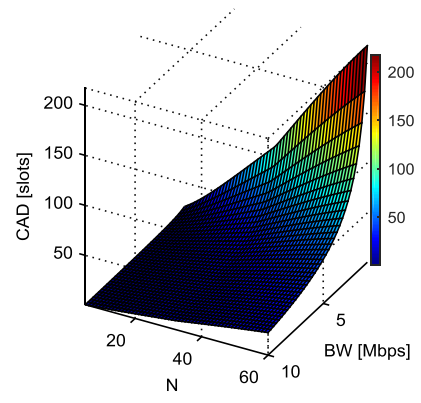

(b)

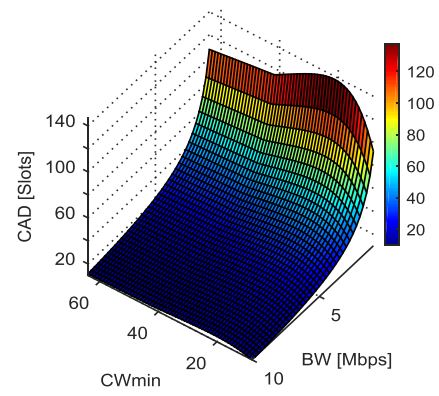

(c)

Fig. 6. Joint impact of varying (a) $N$ and $C W_{\min }$ (b) $\mathrm{N}$ and $\mathrm{BW}$

(c) $B W$ and $C W_{\text {min }}$ on average $C A D$

The result in Fig 6(b) shows the impact of varying $N$ and $B W$ of a channel on $A C A D$ experienced by a vehicular node. Decrease in $A C A D$ with increasing values of $B W$ can be attributed to the fact that the channel capacity increases with increase in $B W$. Hence, data can be transferred quickly and other vehicles gets chance to transmit. $A C A D$ increases with increase in $N$ as previously discussed. The impact of BW and $C W_{\min }$ on $A C A D$ experienced by a vehicle on a channel is demonstrated in Fig. 6(c). It is evident from the results that as the $B W$ of a channel increases, $A C A D$ decreases. In case of $C W_{\text {min }}, A C A D$ first increases with increase in $C W_{\text {min }}$, then it starts decreasing. The lower values of $A C A D$ at smaller $C W_{\min }$ values are caused due to the saturation state of the network. It should be noted that lower values of $A C A D$ for these values of $C W_{\min }$ should not be considered favorable because the model used for estimating $A C A D$ does not account for packet drops in such a state. Once the system comes out of the saturation state, $A C A D$ starts to decrease. 


\section{B. Simulation Results}

In this section, simulations carried out to evaluate the performance of the proposed framework for VCPS environment is discussed focusing on simulation settings, performance metrics, and comparative analysis of results.

1) Simulation Setting

The simulation of the proposed framework for service channel selection in VCPS environment is implemented using network simulator 2 (NS-2). Various classes required for simulation were created and modified in the NS-2 source code. As a simulation area, the real road network of Jawaharlal Nehru University (JNU), New Delhi, India is considered (see Fig $7(a)$ ). The satellite image of JNU road network is retrieved from the Open Street Map (OSM) (see Fig 7(b)). A realistic road traffic scenario is created with the help of the mobility model generator in VANETs (MOVE) and micro traffic simulator known as Simulation of Urban Mobility (SUMO). Theses mobility traffic traces generated are used as input to drive the network simulator. Table II shows the complete list of simulation parameters used to configure the simulation scenario. The two scenarios were considered for measuring the effectiveness of Fuzzy-based channel selection framework. In scenario 1, 30 active source-destination pairs were considered. In scenario 2, active source-destination pairs are increased to 50. The simulation result for every scenario is obtained by averaging results of 25 simulation repetitions with different seeds.

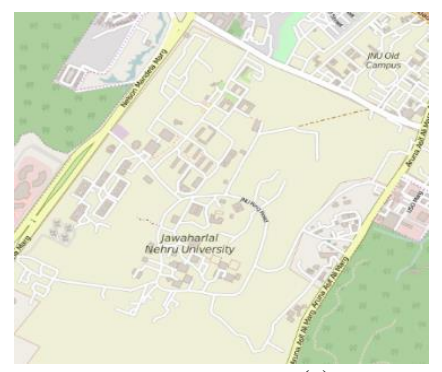

(a)

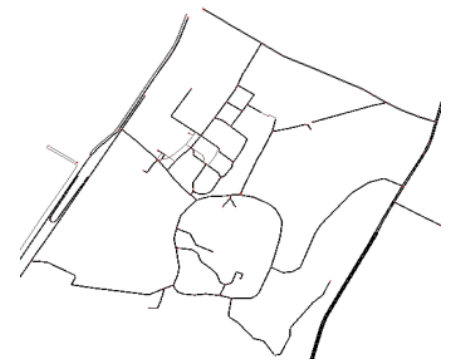

(b)
Fig. 7. The real road network of JNU, New Delhi, India (a) Open Street View (b) Imported view in MOVE

Table II. Simulation parameters

\begin{tabular}{llll}
\hline Parameter & Value & Parameter & Value \\
\hline Area & $1500 \times 1500 \mathrm{~m}^{2}$ & Antenna model & Omni \\
Vehicles & $100-500$ & Phy/Mac & $802.11 \mathrm{p}$ \\
Velocity & $10-100 \mathrm{~km} / \mathrm{hr}$ & Frequency & $5.9 \mathrm{GHz}$ \\
Transmission Range & $300 \mathrm{~m}$ & MAC data rate & $10 \mathrm{Mbps}$ \\
Network traffic & $C B R(512 \mathrm{~B}, 6 \mathrm{pps})$ & Query period & $2.5 \mathrm{sec}$ \\
Protocol & UDP & Hello time-out & $1 \mathrm{sec}$ \\
Source-destination pair & 30,50 & $C W_{\text {min }}$ & 15 \\
Queue length & 50 packets & Retransmission limit & 5 \\
Channel type & Wireless & Simulation time & $1000 \mathrm{sec}$ \\
Propagation Model & Shadowing & & \\
\hline
\end{tabular}

\section{2) Analysis of Results}

The two scenarios are considered for analysis. In the first scenario, thirty active source-destination pairs are considered in a multichannel environment. The result in Fig. 8 shows the PDR of F-CSMV and state-of-the-art protocols with varying density and velocity of vehicles. The PDR of all the considered protocols decreases with increase in velocity and density of vehicles. The decrease in PDR with increase in vehicle's density is due to the fact that more vehicles try to contend for the channel access which increases the number of collisions. With the increase in velocity of vehicles, link failure frequency increases. This leads to loss of more data packets, thereby reducing PDR. It is evident from the results that PDR of FCSMV is higher as compared to other protocols for the considered range of velocity and density of vehicles. This can be attributed to the reason that F-CSMV considered both link quality and channel congestion metric while selecting an SCHs for data transmission. IAR protocol considers SIR metric in the selection of SCH. A channel selection scheme solely focusing on SIR metric may induce vehicles to choose more congested channels. This causes the PDR of IAR to be lower than FCSMV. The PDR of MSCS is the lowest, as it chooses an SCH only on the basis of number of nodes on a channel. It does not consider any channel quality parameter in the selection of $\mathrm{SCH}$.

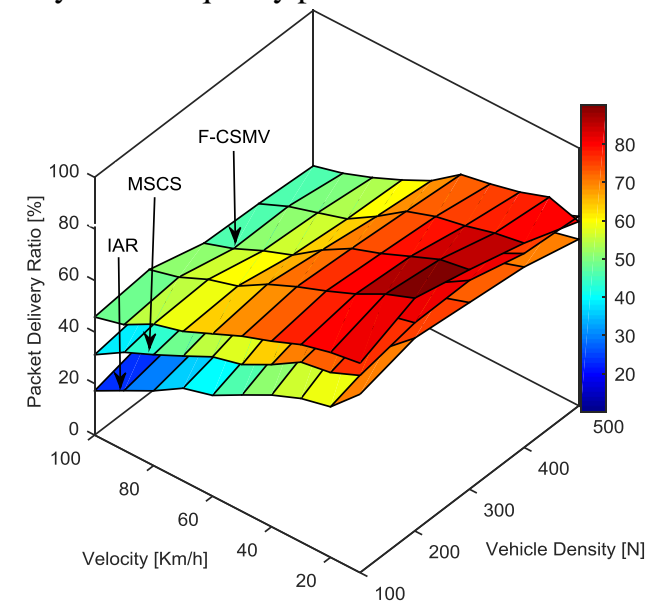

Fig. 8. PDR as a function of vehicle density and velocity

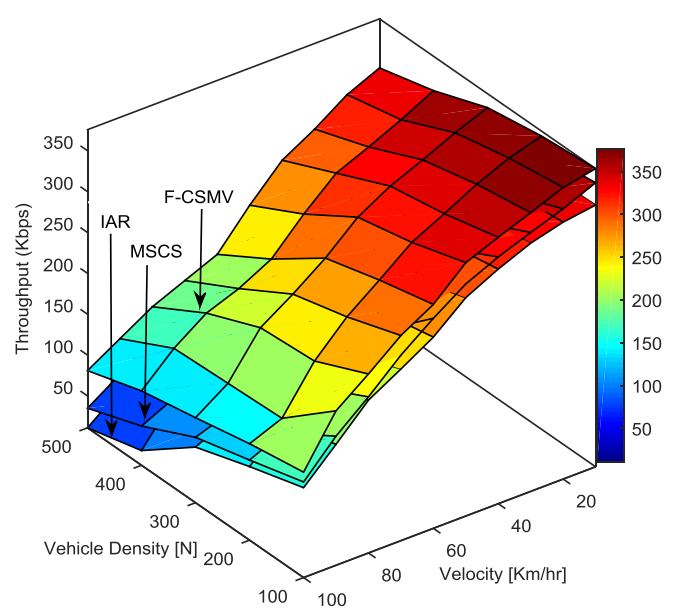

Fig. 9. Throughput as a function of vehicle density and velocity

A comparison of throughput between F-CSMV and state-ofthe-art technique with different vehicle's density and velocity is presented in Fig. 9. It can be observed that F-CSMV achieves higher throughput in comparison to IAR and MSCS protocols for the considered range of velocity and density of vehicles. The higher throughput of F-CSMV can be attributed to two reasons: first, it uses dual transceiver which allows parallel transmissions. Second, the protocol takes into account metrics which depicts channel congestion and link quality in channel selection criteria. This is implemented through a fuzzy-based 
channel selection scheme. A channel having high SIR and low average $\mathrm{CAD}$ is preferred for data transmission, resulting in a higher throughput.

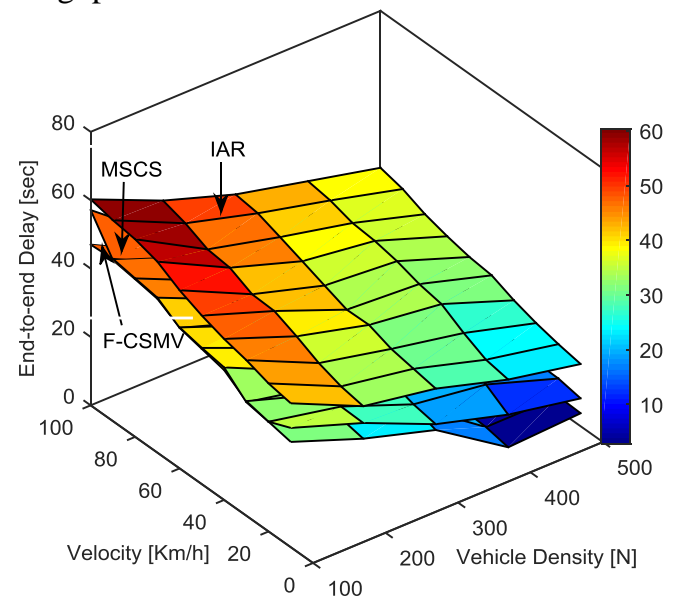

Fig. 10. E2ED as a function of vehicle density and velocity

A comparison of end-to-end delay between F-CSMV and state-of-the-art techniques with varying density and velocity of vehicles is presented in Fig. 10. It is evident from the results that the F-CSMV experiences lower end-to-end delay, as it considers channel quality parameter, namely average $\mathrm{CAD}$, in channel selection criteria. A channel with low average CAD is preferred for sending the data to the destination vehicle in the least time. The higher end-to-end delay of IAR and MCMS is due to usage of congested channels. It ultimately increases the queuing time of the nodes and may even lead to rejection of packets.

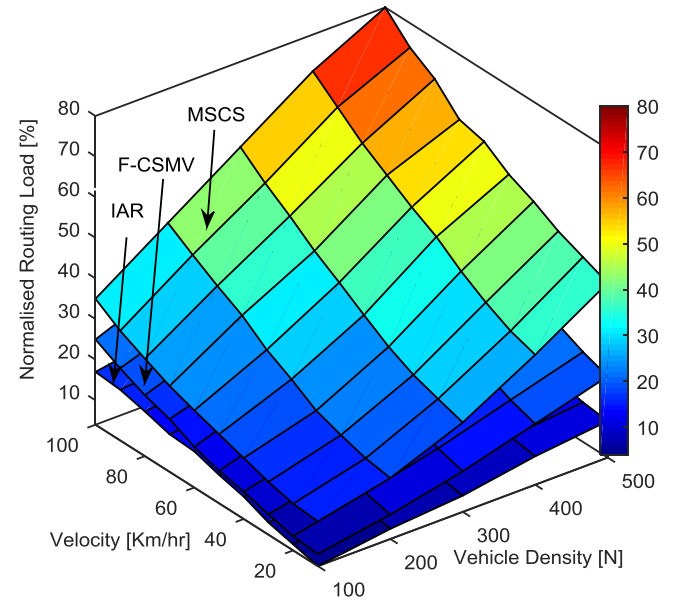

Fig. 11. NRL as a function of vehicle density and velocity

The result in Fig. 11 shows simulation results for normalized routing load with varying vehicle density and velocity of the vehicles. As evident from the results that NRL increases with increase in the velocity and density of vehicles in a network. FCSMV experiences higher NRL than IAR but lower than MSCS. This is because of the fact that F-CSMV utilizes control packets to estimate channel access delay and link quality to use these parameters in channel selection. Although the NRL of FCSMV is higher than IAR, it shows good performance regarding delivery ratio, throughput, and delay.

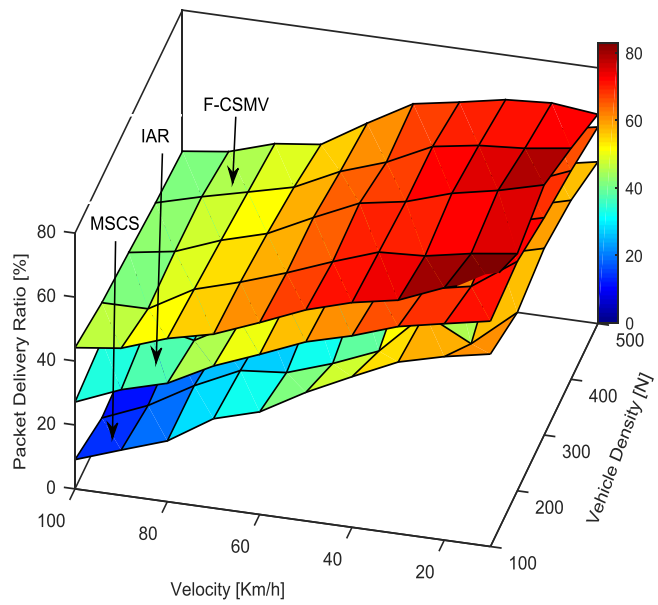

(a)

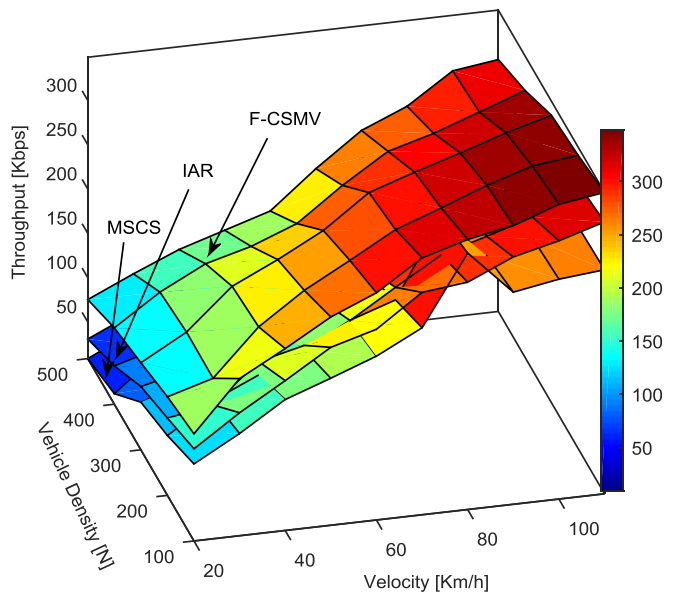

(b)

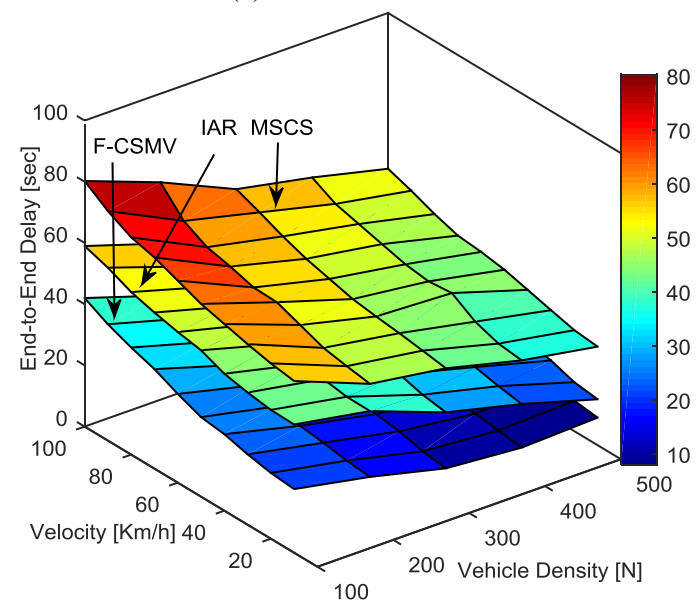

(c)

Fig.12. Joint impact of density and velocity of vehicles on (a) PDR (b) Throughput (c) End-to-end delay

In another testing scenario, fifty source and destination pair is considered for evaluating the percentage difference due to the higher number of network connections along with multichannel vehicular environments. The result in Fig. 12 (a), (b) and (c) shows the joint impact of velocity and density of vehicles on PDR, throughput, and E2ED of F-CSMV and state-of-the-art protocols. Not surprisingly, the pattern of the performance of the F-CSMV and state-of-the-art protocols in scenario 2 is similar to the scenario 1 . The considered protocols performance 
degrade with increase in number of active source-destination pairs. However, each protocol degrades by a different percentage. Approximately 1\% - 5\% degradation is observed in the performance of F-CSMV protocol as compared to scenario 1. For IAR, 5\%-11\% degradation is observed, and 10\%-20\% degradation is observed in the performance of MSCS.

\section{3) Summary of Observations}

The fuzzy based Channel Selection framework for VCPS environment significantly improves the performance of location-oriented services. This framework is developed to estimate channel quality considering signal-to-interference ratio and channel access delay towards optimal channel selection in multichannel vehicular environment. Number of nodes trying to access the channel, min contention window size, and bandwidth of a channel have a considerable impact on the value of $A C A D$ experienced by a vehicle on a channel. ACAD increases with increase in the number of nodes attempting to access the channel. It decreases with increase in bandwidth and min contention window size. The analysis of simulation results in a realistic scenario attests the suitability of the proposed framework in the multi-channel vehicular environment. Although the normalized routing load of the proposed framework is higher than IAR, it shows good performance in terms of packet delivery ratio, throughput and end-to-end delay for the considered range of velocity and density of vehicles. Therefore, the proposed fuzzy based channel selection framework is suitable for dynamic and congestion prone VCPS environments.

\section{CONCLUSION AND FUTURE WORK}

In this paper, a Fuzzy-based Channel Selection framework for location-oriented services in Multichannel Vehicular cyberphysical system environments (F-CSMV) is proposed. The quantification of channel quality considering interference and access delay to select transmission channel reduces the traffic imbalance problem among service channels. The throughput and packet delivery ratio of F-CSMA is higher, and the end-toend delay is lower in comparison to IAR and MSCS in the real map-based road network considering different velocity and density of the vehicles. When the number of source-destination pairs is increased, the degradation in performance of F-CSMA is the least. The proposed fuzzy-based framework is useful for aiding various location-based services in vehicular cyberphysical systems environments. In future research work, authors will consider more channel parameters in dynamic channel selection framework.

\section{REFERENCES}

[1] J. Wan, D. Zhang, S. Zhao, L. Yang, and J. Lloret, "Context-aware vehicular cyber-physical systems with cloud support: Architecture, challenges, and solutions," IEEE Communications Magazine, vol. 52, no. 8, pp. 106-113, 2014.

[2] O. Kaiwartya, A. H. Abdullah, Y. Cao, A. Altameem, M. Prasad, C. T. Lin, and X. Liu, "Internet of Vehicles: Motivation, Layered Architecture, Network Model, Challenges and Future Aspects," IEEE Access, vol. 4, pp. 5356-5373, 2016.

[3] Cao, Y., Wang, N., "Toward Efficient Electric-Vehicle Charging Using VANET-Based Information Dissemination" IEEE Transactions on Vehicular Technology, vol. 66, no. 4, pp.2886-2901, 2017.

[4] Cao, Y., Yang, S., Min, G., Zhang, X., Song, H., Kaiwartya, O. and Aslam, N., "A Cost-Efficient Communication Framework for Battery-Switch-
Based Electric Vehicle Charging", IEEE Communications Magazine, 55(5), pp.162-169", 2017.

[5] Kaiwartya, O., Kumar, S., Lobiyal, D.K., Abdullah, A.H. and Hassan, A.N., "Performance improvement in geographic routing for vehicular Ad Hoc networks", Sensors, 14(12), pp.22342-22371, 2014.

[6] Cao, Y., Sun, Z., Wang, N., Riaz, M., Cruickshank, H., Liu, X., "Geographic-based spray-and-relay (GSaR): an efficient routing scheme for DTNs". IEEE Transactions on Vehicular Technology, 64(4), pp.1548$1564,2015$.

[7] Khedr, W.I., "Improved Lightweight Authentication Scheme for IEEE 802.11p Vehicle-to-Infrastructure Communication" Adhoc \& Sensor Wireless Networks, vol. 31, pp. 227-258, 2016.

[8] D. Jiang, V. Taliwal, A. Meier, W. Holfelder, and R. Herrtwich, "Design of $5.9 \mathrm{GHz}$ DSRC-based vehicular safety communication," IEEE Wireless Communications, vol. 13, no. 5, pp. 36-43, 2006.

[9] "IEEE Standard for Wireless Access in Vehicular Environments (WAVE) - Multi-Channel Operation, IEEE Std. 1609.4-2016,” 2016.

[10]C. Campolo and A. Molinaro, "Multichannel communications in vehicular Ad Hoc networks: a survey," IEEE Communications Magazine, vol. 51, no. May, pp. 158-169, 2013.

[11]P. Fazio, F. De Rango, and C. Sottile, "A Predictive Cross-Layered Interference Management in a Multichannel MAC with Reactive Routing in VANET," IEEE Transactions on Mobile Computing, vol. 15, no. 8, pp. 1850-1862, 2016.

[12]N. Jain, S. R. Das, and N. Asis, "A Multichannel CSMA MAC protocol with Receiver-based Channel Selection for Multihop Wireless Networks," in Tenth International Conference on Computer Communications and Networks, pp. 432-439, 2001.

[13]C. Wu, S. Ohzahata, and T. Kato, "Flexible, Portable, and Practicable Solution for Routing in VANETs: A Fuzzy Constraint Q-Learning Approach," IEEE Transactions on Vehicular Technology, vol. 62, no. 9, pp. 4251-4263, 2013.

[14] Y. Zang, L. Stibor, B. Walke, H. J. Reumerman, and A. Barroso, "Towards Broadband Vehicular Ad-Hoc Networks - The Vehicular Mesh Network (VMESH) MAC Protocol," Wireless Communications and Networking Conference, 2007. WCNC 2007. IEEE, pp. 417-422, 2007.

[15]H. A. Omar, W. Zhuang, and L. Li, "VeMAC: A TDMA-based MAC Protocol for Reliable Broadcast in VANETs," IEEE Transactions on Mobile Computing, vol. 12, no. 9, pp. 1724-1736, 2013.

[16] S. Bharati and W Zhuang, "Performance Analysis of Cooperative ADHOC MAC for Vehicular Networks," pp. 5482-5487, 2012.

[17]B. Tsogoo and S. J. Yoo, "Efficient Channel Management Mechanism to Enhance Channel Utilization for Sensing Data Delivery in Vehicular Ad Hoc Networks," International Journal of Distributed Sensor Networks, vol. $1,2015$.

[18] X. Li, B. J. Hu, H. Chen, G. Andrieux, Y. Wang, and Z. H. Wei, “An RSUCoordinated Synchronous Multi-Channel MAC Scheme for Vehicular Ad Hoc N etworks," IEEE Access, vol. 3, pp. 2794-2802, 2015.

[19] S. Chantaraskul, K. Chaitien, A. Nirapai, and C. Tanwongvarl, "Safety Communication Based Adaptive Multi-channel Assignment for VANETs," Wireless Personal Communications, pp. 1-16, 2015.

[20] S.-L. Wu, C.-Y. Lin, Y.-C. Tseng, and J.-P. Sheu, "A New Multi-Channel MAC Protocol with On-Demand Channel Assignment for Multi-hop Mobile Ad-hoc Networks," Parallel Architectures, Algorithms and Networks, 2000. I-SPAN 2000. Proceedings. International Symposium on, vol. 4, pp. 232-237, 2000.

[21]S.-L. Wu and S.-L. Wu, "A Multi-Channel MAC Protocol with Power Control for Multi-hop Mobile Ad Hoc Networks," The Computer Journal, vol. 45, no. 1, pp. 101-110, 2002.

[22]M. Ni, Z. Zhong, and D. Zhao, "A Novel Multichannel Multiple Access Protocol for Vehicular Ad-hoc Networks," IEEE International Conference on Communications (ICC), pp. 528-532, 2012.

[23]Hang Su and Xi Zhang, "Clustering-Based Multichannel MAC Protocols for QoS Provisionings Over Vehicular Networks," IEEE Transactions on Vehicular Technology, vol. 56, no. 6, pp. 3309-3323, 2007.

[24] M. Boban and A. Festag, "Service-Actuated Multi-Channel Operation for Vehicular Communications," Computer Communications, pp. 1-10, 2015.

[25]Zhao, G., Chen, M., "A Multi-Dimensional Congestion Control Mechanism in Delay Tolerant Networks", Adhoc \& Sensor Wireless Networks, vol. 28, pp. 319-345, 2015.

[26] Felemban, E., Ekici, E.,"Single hop IEEE 802.11 DCF analysis revisited: Accurate modeling of channel access delay and throughput for saturated and unsaturated traffic cases", IEEE Transactions on Wireless Communications, vol. 10, pp.3256-3266, 2011. 


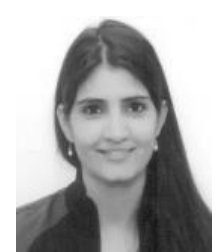

Reena Kasana (M'18) is currently a Ph.D. candidate at the School of Computer and Systems Sciences, Jawaharlal Nehru University, New Delhi, India. He received her MTech degree from the same school in 2013. Her research interest includes Vehicular Adhoc Networks, Wireless Sensor Networks, and Internet of Things.

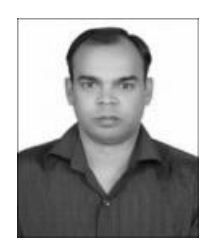

Sushil Kumar (M'14) received his Ph.D. degree in Computer Science from School of Computer and Systems Sciences, Jawaharlal Nehru University, New Delhi, India in 2014. He is currently an Assistant Professor at the Jawaharlal Nehru University, India. His research interest includes Vehicular Ad-hoc Networks, and Wireless Sensor Networks, and Internet of Things.

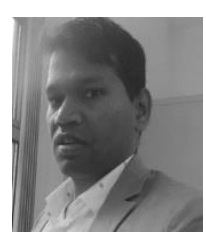

Omprakash Kaiwartya (M'15) received his $\mathrm{Ph} . \mathrm{D}$. degree in Computer Science from School of Computer and Systems Sciences, Jawaharlal Nehru University, New Delhi, India in 2015. He is currently a Research Associate at the Department of Computer and Information Sciences, Northumbria University, UK. He was Postdoctoral Research Fellow at the Faculty of Computing, Universiti Teknologi Malaysia, Johor Bahru, Malaysia. His research interests focus on Internet of connected Vehicles, Electronic Vehicles Charging Management, and IoT use cases in Sensor Networks.

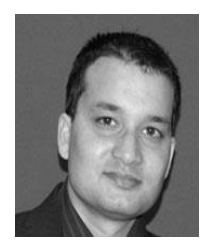

Rupak Kharel (M'17) received his Ph.D. degree in Computer Science from the Department of Computer and Information Sciences, Northumbria University, UK in 2011. $\mathrm{He}$ is currently a Senior Lecturer at the School of Engineering, Manchester Metropolitan University, UK. His research interests focus on Cyber Security oriented technical advancements for Internet of Things, Wireless Sensor Networks, and Industry 4.0.

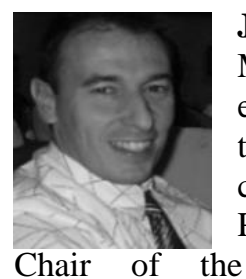

Jaime Lloret (M'07, SM'10) received his M.Sc. in Physics in 1997, his M.Sc. in electronic Engineering in 2003 and his Ph.D. in telecommunication engineering in 2006. He is currently Associate Professor at the Universitat Politecnica de Valencia, Spain. He was the Chair of the Internet Technical Committee (IEEE Communications Society and Internet society) and Working Group of the Standard IEEE 1907.1. He has authored 22 book chapters and has more than 340 research papers published in national and international conferences, international journals (more than 110 with ISI). He has been the co-editor of 38 conference proceedings and guest editor of several international books and journals. He is editor-in-chief of the Ad Hoc and Sensor Wireless Networks, and associate editor of 46 international journals (16 ISI). He has been general chair of 28 International workshops and conferences.

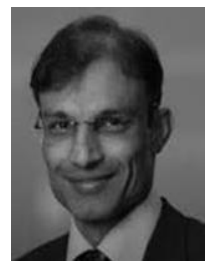

Nauman Aslam (M) received his Ph.D. in engineering mathematics from Dalhousie University, Canada, in 2008. He is currently a Reader (Associate Professor) in the Department of Computer and Information Sciences, Northumbria University, UK. He was an assistant professor at Dalhousie University from 2008 to 2011. He has extensive research experience in wireless ad-hoc and sensor networks.

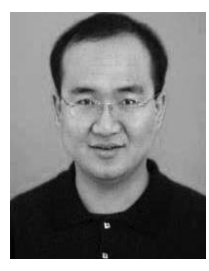

Wang Tong is an Associate Professor at Information and Communication Engineering College, Harbin Engineering University, China. $\mathrm{He}$ received $\mathrm{PhD}$ degree in Computer Application from Harbin Engineering University in 2006. His research interests include Wireless Sensor Networks, Vehicular Ad-Hoc Networks and Internet of Things. 